\title{
A Research on User Interest Model Using Ontology and VSM Extension
}

\author{
Yue-zhong $\mathrm{Wu}^{1,2}$ and Shu-hong $\mathrm{Chen}^{3}$ \\ ${ }^{1}$ School of Information Science and Engineering, Central South University, \\ Changsha 410083, China \\ ${ }^{2}$ College of Computer and Communication, Hunan University of Technology, \\ Zhuzhou 412007, China \\ ${ }^{3}$ School of Computer and Communication, Hunan Institute of Engineering, \\ Xiangtan 411104, China \\ 8986259@qq.com
}

\begin{abstract}
The user interest model is the key technology for us to efficiently obtain personalized high quality resources in huge amounts of data. On the basis of traditional user interest model, combining with the concept of ontology, this paper comes up with a construction method based on ontology semantic tree and space vector method, and its eigenvalue is changing by using forgetting curve. The model describes a theme by using semantic concept tree, and it not only simply to describe the semantic relations between concepts, but also can reflect the user interest drift character. It is more accurate in characterization of user interest, and better reflects the change of user interest, and efficiently improves the quality of network document resource personalized services. Finally the experiment describes the update process of the proposed model by using an example, and illustrates that the proposed model is simple and practical.
\end{abstract}

Keywords: user interest model; ontology; space vector method; forgetting curve

\section{Introduction}

In an open network environment, the data that people use and make in the study, work and life is increasing in the way of geometric progression. Network document resources is viewed as a kind of special resources of the Internet and its rapid growth makes the "information overload" and "resources trek" become more and more serious, which has seriously affected people access to information efficiently. Simultaneously appearance of mass and excess information, makes the user can not easily get the resources they need quickly and precisely. In the existing solutions, whether it is Google, Baidu and other search platform or a variety of intelligent recommendation system [1-5], the key technology to achieve the high quality of the user's personalized service is the structure of the user interest model. The user interest model is a data model which reflects the user interest feature, in other words, it is a computer language describing the user's informative need and it is the direct object of the personalized information service. With the user interest model, meta-search engine can accordingly actively service and provide personalized search service for different users. So the user interest model is the foundation and core of personalized service, the quality of user interest model is directly related to the quality of personalized service [6].

Based on the traditional model of the user's interest, combined with the concept of ontology, we propose a method to build the vector space model based on user interest ontology semantic tree. The model can describe a topic with semantic 
concept tree, and it is different from the traditional method based on keywords description, and the model can describe the simple semantic relations between concepts. The main contributions are as follows:

- In order to improve the accuracy of the user to obtain network documents, we proposed an extended VSM user interest model based on ontology, which is more accurate.

- By introducing the forgetting function based on the Ebbinghaus curve, it can be adapted to the user's interest drift character and provide accurate personalized service.

- We designed a complete user interest model, which includes the representation, updating and feedback mechanism. The method is simple and practical.

- We did the experimental simulation and the results show that the user interest model can accurately reflect the user's interest and its ability to update and expand is stronger.

The remainder of this paper is organized as follows: Section 2 surveys related work in the literature and presents our approach. Section 3 describes the overview of the extended VSM user interest model based on Ontology. Sections 4 describe the construction of user interest model. The experimental design shows the numerical results that are obtained through the update of our proposed model. Finally, Section 5 concludes this paper and suggests future work.

\section{Related Work}

\subsection{User Interest Model}

The research of user interest model is concentrated in paper [7]. Theme representation is that using the contents and themes which users are interested in to represent user interest model; Keywords representation method is using the keywords of user interest information to express the user interest model; User's bookmarks are represented by a user's interest model by means of an important site or page's bookmark. The representation of Space Model Vector (VSM) is used to represent the user interest model in the vector space; A representation method based on the neural network is that using the network state linking weight feature to represent the user interest model; Based on the user, the representation of the project evaluation matrix uses a two-dimensional matrix to express the user interest model; Ontology representation method uses ontology to express the user interest theme and realizes the reuse and share of knowledge; Interest expression method, according to the user interest information granularity to represent the user interest model, is divided into coarse interest granularity and fine granularity representation method, whether interested in the coarse granularity, to distinguish between user interest theme and content, etc. In Hao Shuilong and others papers [8], they proposed the use of hierarchical vector space model to model the user interest, and based on the theme and its feature to form the user interest to improve the accuracy and practicality of the user interest model. In this paper, based on the vector space model, combined with domain ontology, a VSM user interest model based on semantic tree is constructed, which can be adapted to the user's interest and interest migration, and provide accurate personalized service.

\subsection{Ontology}

Ontology is a formal, shared and clear specification of the conceptualization [9]. It is a description of the concepts and relations, through the standardization of the concepts and their relations to describe the basic knowledge system in a certain field. Generally speaking, an ontology can be composed of five elements, the 
concept, the relation, the function, the axiom and the instance [10]. Domain ontology studies how to define the concept of a specific domain, the relationship between concepts, the activity and the main theory and the basic principle of the field. From the construction of user interest model to the application domain in the literature [11-13],they can reflect the user's interest and provide personalized service. This article is aimed to the network document resources, using catalog information of Chinese book classification method as domain ontology.

\subsection{Forgetting Function}

People's memory will gradually disappear as time goes by, and when some of the memory information in the human brain is not used, the information will be lost. But when some information is reemerging, the relevant information in the human brain will gradually recover. Day after day, the important information which is repeated and reinforced will be retained. According to the memory theory, we can suppose that the change of user interest is a kind of memory forgetting phenomenon. German psychologist Hermann Ebbinghaus thinks that the speed of forgetting is the fastest after we just remembering, and then its slow down, until the end of the process of forgetting. Zhang Gewei and others [14] came up with a method, through the establishment of the memory of the rules of the law of memory - forgetting the mathematical model, applying to knowledge management, and extracting effective knowledge. By using the power function curve or normal distribution density curve in the literatures [15-16], it is found that building short term and long term interest model, accurately identifying and tracking the user's interest changes can solve the problem of user interest drift. Based on Ebbinghaus's theory of forgetting, we use it to reduce the weight of interest value, and update the user interest model.

\section{Extended VSM User Interest Model Based on Ontology}

\subsection{Model Frame}

This model is applied to the development of the network document sharing application system [17] based on cloud storage. Based on the spatial vector representation of user interest model, combining domain ontology and Ebbinghaus forgetting curve, we construct an extended VSM user interest model based on ontology which include Input Layer, Processing Layer and Output Layer which is as shown in Figure 1.

Input Layer is a data acquisition part. It is mainly the acquisition of user interest related information. We use both manual and automatic acquisition mode to the users' interest. Manual way is through the user to add the key words of interest and the automatic method is to deal with the user's access record and download the document to obtain the concept keywords and the main feature keywords. The realization of adaptive update in the user interaction with the system in the process and these words are divided into Words Library.

Processing Layer is a core part of the data processing. It obtains the specification data by preprocessing, and then obtains the user interest ontology with the domain ontology, and returns the related data in the process of storage by the feedback mechanism. Through the pretreatment, the construction of the user interest ontology and the change of the interest of forgetting functions, Processing Layer is to analyse and handle.

Output Layer is the representation and update of user interest model, which is directly used to search and recommend the network document sharing application system, at the same time, to feedback the updated user interest model to Input Layer and Processing Layer. 


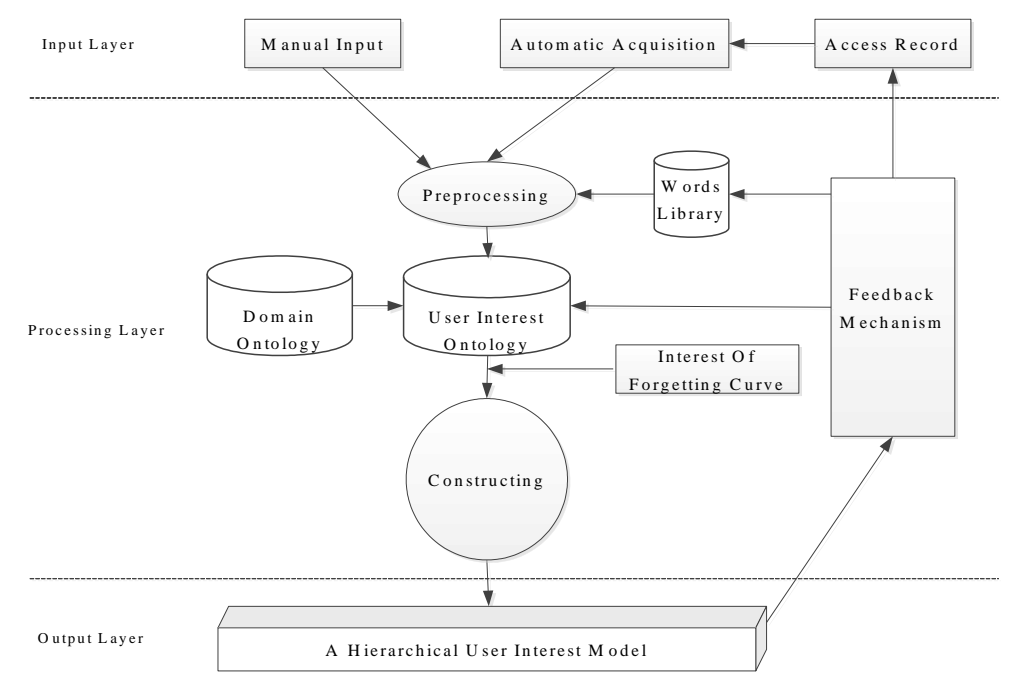

Figure 1. Extended VSM User Interest Model Based on Ontology

\subsection{Related Concepts}

In the user interest model presented in this paper, some concepts are related to the following.

1) Network document

The network document refers to a piece of article. Its format not only can be structured types such as txt and XML, also can be unstructured types such as word and pdf. First, the system uses Lucene to segment the source document, create index, and then upload it to the system storage according to the theme classification. Network document is represented as a document vector $d:\left\{\left(t_{l}, w_{l}\right), \ldots,\left(t_{n}, w_{n}\right)\right\}$, where $t_{i}$ represents the document feature item keywords and $w_{i}$ represents the important degree of the feature item $i$ keywords in document $d$.

Document feature item keywords weight using TF-IDF method in Eq.(1):

$W_{t, d}=t f_{t, d} \times \log \left(n / d f_{t}\right)$

Among them, $W_{t, d}$ represents the weight of the keywords $t$ in document $d$, and $t f_{t, d}$ represents the frequency of the keywords $t$ in document $d$, and $\mathrm{n}$ represents the total number of documents in the document set $D, d f_{t}$ represents the number of documents containing keywords $t$.

2) Domain ontology

Combined with the specific application scenarios, this paper will be represented as a three tuple: $D O=(I, C, R)$

$I$ is the domain ontology basic information, including the name of the ontology, the creator, the creation time, the application domain and so on; $C$ is the collection of the related concepts in the domain ontology, and $R$ is a set of relations among the domain ontology.

In this system, the construction of its ontology domain directly uses the directory information of Chinese book classification method. There are three kinds of relations between the concept of Ontology: inheritance relationship which is represented by the words "subclass of" means that the concept is the relationship between the parent and the child; synonymous relationship which is represented by the words "synonymy of" means that it is equal between concepts; attribute relationship which is represented by the words "attribute of" means that a concept is the relationship to describe another. 
For example: $D O_{1}=\left(I_{1},\left\langle c_{1}, c_{2}, c_{3}, c_{4}\right\rangle,\left\langle r_{1}, r_{2}, r_{3}\right\rangle\right)$

$r_{1}=$ kind of $\left(c_{1}, c_{2}\right), r_{2}=\operatorname{subclass}$ of $\left(c_{2}, c_{3}\right), r_{3}=$ synonymy of $\left(c_{3}, c_{4}\right)$

3) User interest Ontology

In the system, we have a set of users $U=\left\{u_{1}, \ldots, u_{n}\right\}$, and each user has his own hobby UI, The user interest model is represented by vector space model, and the weight that reflecting these features for the user's interest in the important degree is represented by TF.

Combining domain ontology, user interest ontology is represented as a two tuple: $U I O=(D O, U I)$

\section{4) Forgetting function}

Jiang Zhiheng etc. according to the Ebbinghaus forgetting curve propose the following forgetting function[18] in Eq.(2):

$$
J(t)=20 e^{b /\left(t+t_{0}\right)^{c}}
$$

$t$ is as independent variable and $e=2.71828$ is the natural logarithm base, $b=0.42$, $c=0.225, t_{0}=0.00255$

The forgetting mechanism includes explicit and implicit forgetting. When the concept of the user set the value of the time, such as the concept of the existence of time longer than the user defined time ( 2 years), then occurre a explicit forgetting; When the system considers that a concept has nothing to do with the user (this paper refers to the concept of the user's interest in the ontology of the user's interest), then occurre a hidden type of memory.

\subsection{Model Building Algorithm}

\subsubsection{Modeling:}

\section{1) Express}

The user interest vector space model is represented by a hierarchical model. Coarse- grain classification is carried out by using the top level topics. And give a theme to have a simple relationship between the feature keywords. But the concept keywords between the theme and the feature keywords, and the relationship between the three, are described by the user interest Ontology.

$$
\left\{\left[T_{1},\left(K_{11}, W_{11}\right),\left(K_{12}, W_{12}\right), \ldots,\left(K_{l h}, W_{l h}\right)\right], \ldots,\left[T_{i},\left(K_{i j}, W_{i j}\right)\right], \ldots\left[T_{m},\left(K_{m n}, W_{m n}\right)\right]\right\}
$$

$T_{i}$ represents the theme $i . K_{i j}$ represents the feature $j$ keywords of the theme $i . W_{i j}$ represents the weight of the feature $j$ keywords in the theme $i$ in Eq.(3), $T, K \in C$.

$$
W_{i, j}=\frac{t_{i, j}}{f_{j}}
$$

$W_{i, j}$ represents the weight of $K_{i j}$ in the user interest model vector $U I . t_{i, j}$ represents the number of $K_{i j}$ that appears in the user interest model vector $U I . f_{j}$ represents the number of the keywords that appears at most in the user interest model vector $U I$.

\section{2) Initialization}

The user interest model need to be initialized for the first time to use. We use two ways to initialize. We set the value range of interest $[0,1]$, with each interest in the domain ontology library, an interest value which is the weight of feature keywords is given. When the value is close to 1 , it shows that the greater the user interest.

User involvement: according to the user manual input the feature keywords, the corresponding frequency is 1 , then the weight is equal. 
User does not participate in the way: through the user's continuous use of the model and the system feedback mechanism, dynamic automatically update the weight of the relevant interest.

3.3.2. Update: Based on the study of the law of human brain memory in cognitive science and according to the properties of forgotten memories, the paper proposes a method of user interest model updating based on forgetting function. On the one hand, it is consistent with inheritance and property relations and addresses the same theme's interest. On the other hand, it accords with the synonymous relationship and solves the synonymy problem.

Through analysis, we find that user interest will have the following four situations: interest, stability, forgetting, activation. These are the following three changes in interest: add new interest, delete the interest of forgetting, and update the changes of interest.

We use the user interest ontology to carry out the knowledge management of user interest, combined with the rule of human brain memory, as long as the user's interest are triggered by the user will be retained, but the end of the process and update will get a stable user interest model.

User interest in this experience automatically according to the relationship between the ontology, the ontology is parsed into a semantic tree, and then automatically based on the semantic tree for each concept assignment. We need to follow the following rules in the update.

(1) The relationship between the upper and lower nodes in the semantic tree: Attribute relationship, the upper concept contains $n$ classification, and its dynamic change is according to the overall classification. The actual amount of the underlying concept is $j$.

(2) Upper concept weight calculation: we use arithmetic mean, which is shown in Eq.(4).

$$
W_{i, j}=\frac{1}{n} \sum_{n=1}^{j} w_{i, j}
$$

(3) There are two ways to calculate the weight $W_{i, j}$ of the user interest feature keywords:

i) Feature keywords using Eq.(3);

ii) Concept keywords using Eq.(4).

Based on the above analysis, the user interest model updating algorithm based on forgetting function is described as follows:

\footnotetext{
Input:

$U=\left\{u_{1}, \ldots, u_{n}\right\}, D O=(I, C, R), J(t)$

Output:

UI, UIO

I/If the feature keywords have be accessed more than two years, activated, and initialize $t_{s}$

if $t_{s}>2$ years, Initialization $t_{s}=0$

else

//Interest degree is higher: the feature keywords have be accessed within 7 days

CASE 1: $t_{s} \leq 7$ days, computing by Eq.(3)

//Using stability calculation formula for correction: the feature keywords have be accessed between the 8th day and 30th day

CASE 2: 8 days $\leq t_{s} \leq 30$ days, computing by $0.8 \times E q$. (3)

// Reduce the value according to forgetting function: the feature keywords have be
} 
accessed between the 31 th day and 2 years

CASE 3: 31 days $\leq t_{s} \leq 2$ years, computing by $J(t) \times E q$.(3)

//Performing to forget: the feature keywords have not be accessed more than 2 years

CASE 4: $t_{s}>2$ years, delete the feature keywords and its weight

end if

// calculating the weight of concept keywords

Computing by Eq.(4)

//Regularly updating the user interest model; when the system thinks interest has nothing to do with the user, performing the implicit forgotten

Updating UI, UIO

\section{Construction of User Interest Model}

Here we illustrate the process of constructing a user interest model with an example.

1) Domain ontology construction

Comprehensive consideration, we use OWL language as the ontology modeling language, and the use of Protégé which provides a simple and easy to use graphical interface, a good scalability of a large number of plug-in applications and support rich ontology description language and the integration of knowledge and ontology storage to build the ontology. Using Jena2 to build ontology model to deal with ontology. Jena is an open source semantic web development kit based on Java, which provides an integrated environment for the analysis of RDF, RDFS and OWL ontology, which is equipped with a sound to resolve, storage, query and rule based reasoning engine.

We use Protégé to construct a domain ontology of "Computing Technology, Computer Technology" which is as shown in Figure 2. The domain ontology is constructed as shown in Figure 3.

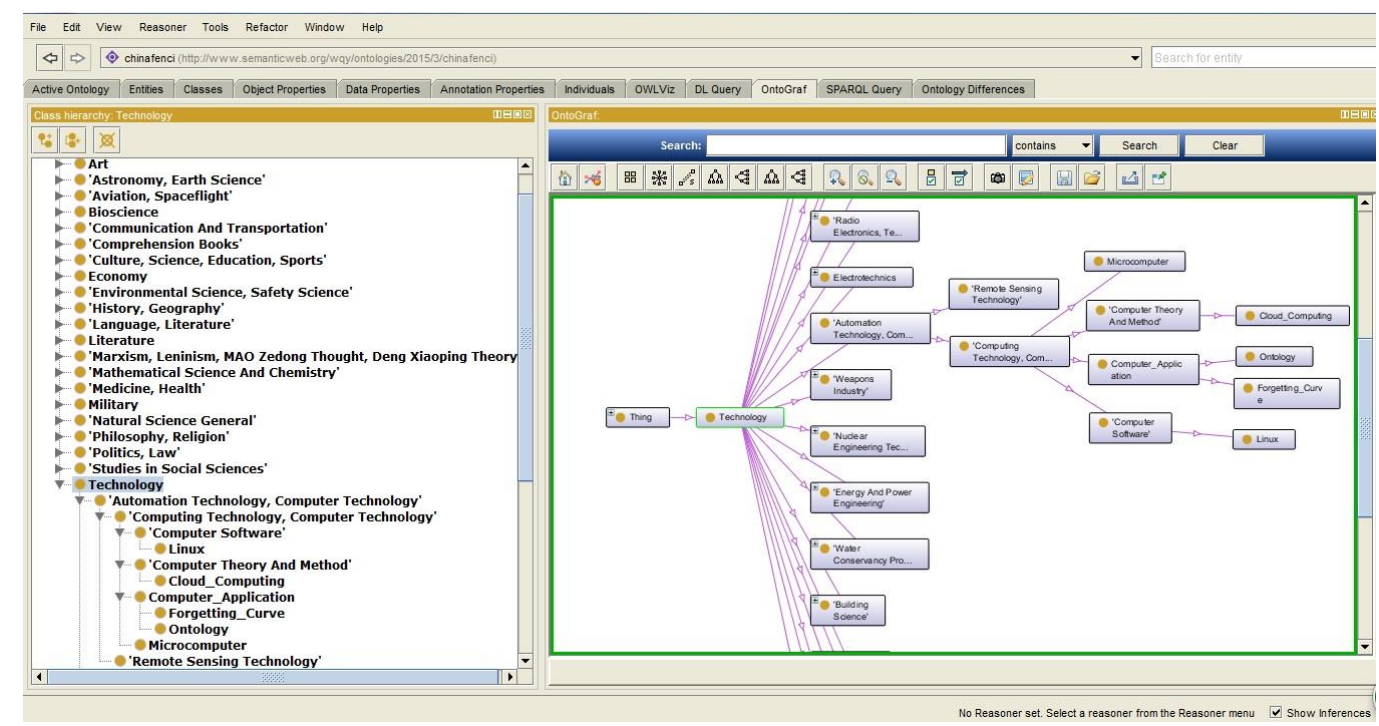

Figure 2. Protégé Building Domain Ontology 


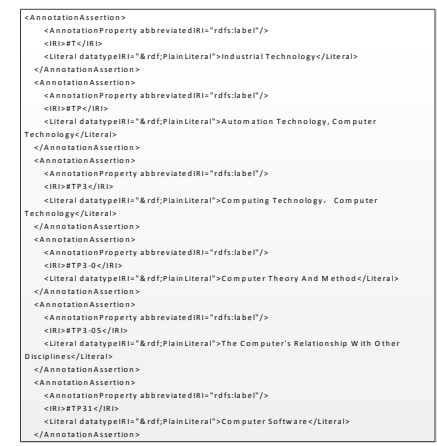

Figure 3. Domain Ontology Construction

2) An extended VSM user interest model based on Ontology

Suppose a user's user interest model $U I=\{[$ "Industrial Technology", (Cloud Computing, 0.8), (Linux, 0.3), (Ontology, 0.5), (Ontology(Chinese), 0.5), (Forgetting Curve, 0.4), (Satellite Remote Sensing, 0.1), (Sensor, 0.2), ["Philosophy, Religion", (Ontology, 0.2), (Ontology(Chinese), 0.2), (Cosmology, 0.1 ), (Forgetting Curve, 0.3)] , a user interest model based on ontology is generated by combining the domain ontology of Chinese book classification. It is as shown in Figure 4.

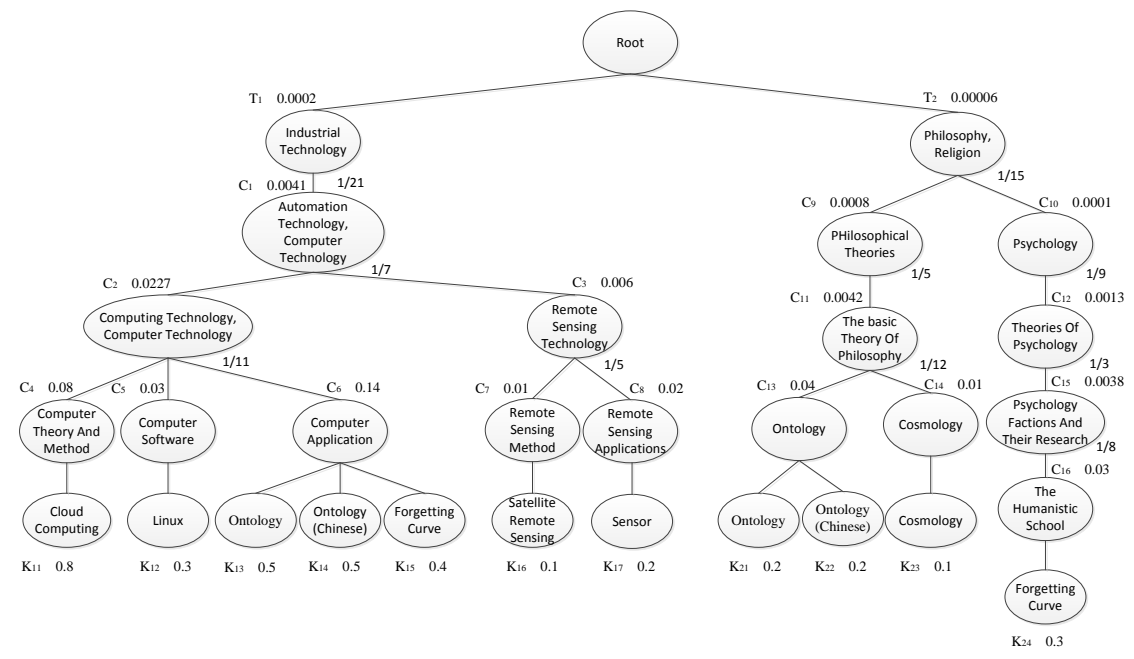

Figure 4. User Interest Model Based on Ontology

In the picture above, using the Chinese book classification method, the classification of the concept class is shown in the graph. For example, the concept of industrial technology contains 21 sub categories, expressed as 1/21; In the example, assume that each concept class contains 10 keywords. Due to more concept, so it gives an example to calculate the weight of the concept keywords:

$$
W\left(C_{6}\right)=1 / 10 *\left(W\left(K_{13}\right)+W\left(K_{14}\right)+W\left(K_{15}\right)\right)=0.14
$$

3) Update of the user interest model

Now suppose that user interest changed as follows: the interest degree of feature keywords Ontology and Ontology(Chinese) rise to 0.8 from 0.5 , feature keywords Linux is accessed within a month, feature keywords Satellite remote sensing and sensor are only two months to visit, feature keywords Cosmology is never visited in 
two years and other feature keywords are access in 7 days. Then it triggers the user interest model update process.

(1) Changing the feature keywords interest degree

$W\left(K_{12}\right)=0.8 * 0.3=0.24$

$W\left(K_{13}\right)=W\left(K_{14}\right)=0.8$

$W\left(K_{16}\right)=20 \times 2.71828^{0.42 /(60+0.00255)^{0.225}} \% \quad * 0.1=0.2364 * 0.1=0.02364$

$W\left(K_{17}\right)=20 \times 2.71828^{0.42 /(60+0.00255)^{0.25}} \% * 0.2=0.2364 * 0.2=0.04728$

(2) Changing the concept keywords interest degree

For example: $W\left(C_{6}\right)=1 / 10 *\left(W\left(K_{13}\right)+W\left(K_{14}\right)+W\left(K_{15}\right)\right)=0.2$

The user interest model updated $U I=\{[$ "Industrial Technology”, (Cloud Computing, 0.8), (Linux, 0.24), (Ontology, 0.8), (Ontology(Chinese), 0.8), (Forgetting Curve, 0.4), (Satellite Remote Sensor, 0.0236), (Sensor, 0.0473)], ["Philosophy, Religion", (Ontology, 0.2), (Ontology(Chinese), 0.2), (Forgetting Curve, 0.3)]\}. The user interest model based on ontology is as shown in Figure 5 and the change of the degree of interest is marked in overstriking.

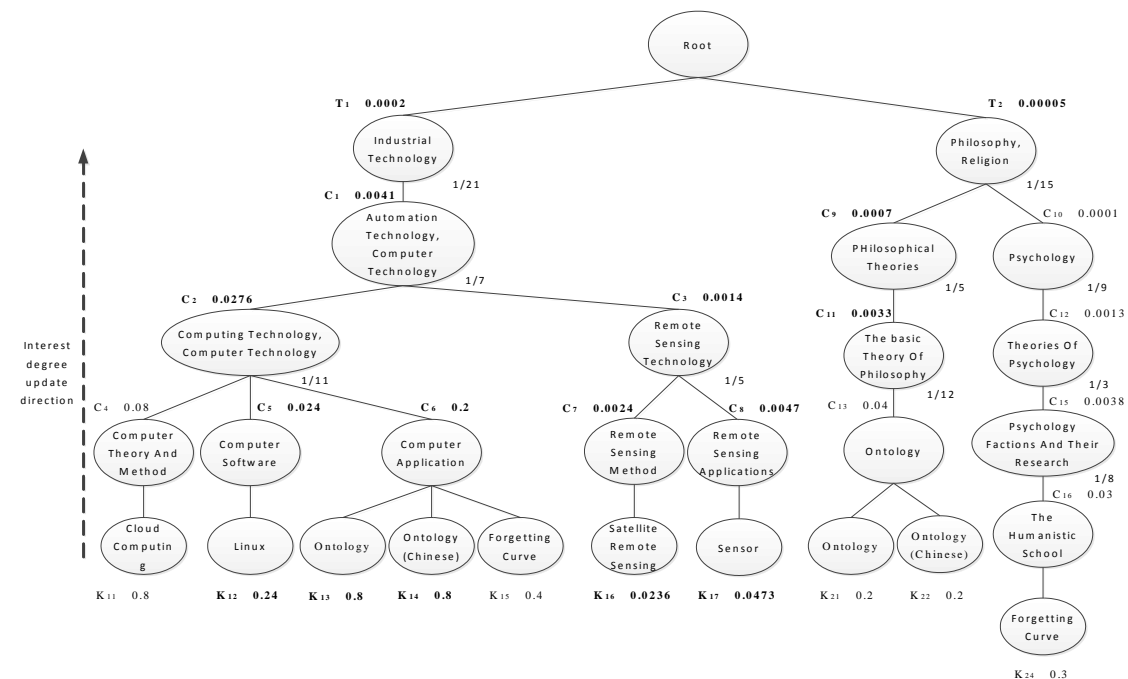

Figure 5. An updated Ontology Based user Interest Model

\section{Conclusion}

In this paper, a VSM user interest model based on ontology extension is proposed, which is based on the user interest ontology with a semantic tree relationship. It uses the forgetting function based on Ebbinghaus's theory of forgetting to update the user's interest, which has a high efficiency. In the future work, we can continue to refine the user interest ontology and analyze the semantic tree, then generate a more reasonable semantic tree structure.

\section{Acknowledgments}

This work is supported in part by the National Natural Science Foundation of China under grant numbers 61502163, 61472451, 61402161, 61350011 and 61379058.

\section{References}

[1] J. B. Schafer, J. Konstan and J. Riedl, "Recommender systems in ecommerce", Proc. of the 1st ACM Conf . on Electronic Commerce, New York: ACM press, (1999).

[2] J Riedl and P. Dourish, "Introduction to the Special Section on Recommender Systems", ACM Transactions on Computer-Human Interaction, vol. 12, (2005).

[3] F. Ricci and H. Werthner, "Introduction to the special issue: Recommender systems", International Journal of Electronic Commerce, vol. 2, no. 1, (2006). 
[4] H. L. Xu, X. Wu, X. D. Li and B. P. Yan, "Comparison Study of Internet Recommendation System", Journal of Software, (In Chinese), vol. 2, no. 20, (2009).

[5] X. W. Meng, H. Xun, L. V. Wang and Y. J. Zhang, "Mobile Recommender Systems and Their Applications", Journal of Software, (In Chinese), vol. 1, no. 24, (2013).

[6] Q. R. Wang, H. Y. Zhao and J. Cao, "User Modeling in Personalized Service Offering", Journal of Chinese Computer Systems, (In Chinese), vol. 1, no. 32, (2011), pp. 39-46.

[7] Q. Liu, "A Study of Designing and Applying Recommenders Based on User Interests Modeling", University of Science and Technology of China, (In Chinese), (2013).

[8] S. L. Hao, G. Q. Wu and X. G. Hu, "Presentation and updation for user profile based on hierarchical vector space model", Journal of Nanjing University (Natural Sciences), (In Chinese), vol. 2, no. 48, (2012).

[9] Z. H. Deng, S. W. Tang, M. Zhang, D. Q. Yang and J. Chen, "Overview of Ontology", Acta Scientiarum Naturalium Universitatis Pekinensis, (In Chinese), vol. 5, no. 38, (2002).

[10] G. Fang and C. Cun-Gen, "Ontology Research and Existing Problems in Knowledge Engineering", Computer Science, (In Chinese), vol. 10, no. 31, (2004).

[11] F. Z. Zhang, W. J. Li and C. Y. Zhu, "User Model for Cross System Personalization Service Based on Domain Ontology", Computer Engineering, (In Chinese), vol. 13, no. 35, (2009).

[12] J. W. Yan, X. Huang, M. Liu, Y. B. Zhu and H. B. Ni, "Personalized recommendation algorithm for user interest model based on ontology", Computer Integrated Manufacturing System, (In Chinese), vol. 12, no. $16,(2010)$.

[13] Y. F. Chen, H. K. Zhao, X. Q. Yu and W. G. Wan, "Research on Ontology-based User Interest Model Construction, Computer Engineering, (In Chinese), vol. 21, no. 36, (2010).

[14] G. W. Zhang, W. H. Liao, C. Y. Liu and F. Yu "Memorizing-Forgetting Featured Knowledge Management and Optimizing Model", Journal of Nanjing University of Aeronautics \& Astronautics, (In Chinese), vol. 2, no. 40, (2008).

[15] H. Yu and Z. Y. Li, "A Collaborative Filtering Recommendation Algorithm Based on Forgetting Curve", Journal of Nanjing University (Natural Sciences), (In Chinese), vol. 5, no. 46, (2010).

[16] X. M. Guo and G. Z. Yi, “A Hybrid Algorithm to Track Drift of User 's Interests”, CAAI Transactions on Intelligent Systems, (In Chinese), vol. 2, no. 5, (2010).

[17] Y. Z. Wu, Q. Liu, C. Y. Li and G. J. Wang, "Research on Cloud Storage based Network Document Sharing", Journal of Chinese Computer Systems, (In Chinese), vol. 1, no. 36, (2015).

[18] Z. H. Jiang and N. Q. Liu, "Theory of Forgetting Function-Discussion About Memory Psychology of Mathematics", Psychology Dynamic, (In Chinese), vol. 3, (1988).

\section{Authors}

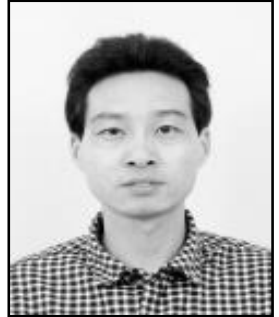

Yuezhong Wu, received the Bachelor's degree in Computer Science from Hunan University of Technology, PR China, in 2004; and the Master's degree in Computer Application Technology from Hunan University of Technology, PR China, in 2007. He has been a Ph.D. candidate at Central South University since September 2009. He research interests include user interest model, recommendation system, intelligent search and cloud computing. He is a member of CCF.

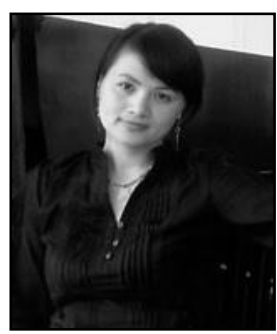

Shuhong Chen, received her Ph.D in Computer Science from Central South University, PR China, in 2014. She is currently an associate Professor in School of Computer and Communication, Hunan Institute of Engineering, China. Her research interests include trust evaluation models and algorithms, mobile social networks, performance analysis, computer networks. 